\title{
Probing Fractional Josephson Junction with a Quantum Dot
}

\author{
P. STEFAŃSKI*
}

Institute of Molecular Physics of the Polish Academy of Sciences, M. Smoluchowskiego 17, 60-179 Poznań, Poland

\begin{abstract}
We consider theoretically a junction between two topological superconducting wires, mediated by a quantum dot. The wires are modelled by the Kitaev chains tuned into topological phase, which possess unpaired Majorana states at their ends. We derive the low energy Hamiltonian of the model. The Majorana states closer to the dot convert into the Dirac fermion inside the dot, forming fractional Josephson junction. The dot is additionally weakly coupled to the normal tunneling probe allowing transport measurement through the dot. When the topological wires are short, the unpaired Majorana end-states can hybridize inside the wire forming an extended Dirac fermionic state. It yields the destruction of the extended state in the dot. We discuss the dependence of the spectral density of the dot and its conductance on superconducting phase. We show that the conservation of parity of the junction, crucial for successful measurement of the fractional effect, can be assured by the gate voltage manipulation of the dot level position and that in case of an unpaired Majorana state in the junction a half conductance quantum can be observed.
\end{abstract}

DOI: 10.12693/APhysPolA.130.554

PACS/topics: 73.63.-b, 73.21.La, 74.78.Na, 74.50.+r

\section{Introduction}

The Majorana fermions, exotic quasiparticles which are their own antiparticles, were proposed by Majorana in the context of particle physics [1]. They are exciting due to both their fundamental properties and potential application in topologically robust quantum computing [2]. The most promising scalable system in solid state appears to be a one dimensional $p$-type superconducting wire, originating from the idea proposed by $\mathrm{Fu}$ and Kane [3]. To make quantum computing on Majorana states feasible one needs methods for their unambiguous identification. One method is measurement of transport characteristics of the tunnel junctions between normal electrode and topological superconducting wire hosting Majorana bound states (MBS) [4-6]. However, there are still discouraging ambiguities whether the observed zero-bias anomaly in differential conductance is caused by Majorana zero modes or has another origin $[7,8]$.

In this work we concentrate on another kind of measurement, which can prove the MBS existence, namely the phase sensitive current measurement in superconducting junctions. When two conventional superconductors are coupled together, they form the Josephson junction. The phase-biased supercurrent through such a junction has a characteristic dependence on the superconducting phase difference: $J \sim \sin (\Delta \phi)$ with $2 \pi$ periodicity. Consequently, the ac Josephson effect has a resonant frequency $\omega_{r}=q V / \hbar$, where $V$ is the applied dc voltage, $\hbar$ is the Planck constant and the charge, $q=2 e$, is twice of electron charge. It is due to the fact that the Cooper pairs tunnel through the junction. This picture changes when conventional superconductors are replaced by topological ones with unpaired MBS at their ends.

*e-mail: piotrs@ifmpan.poznan.pl

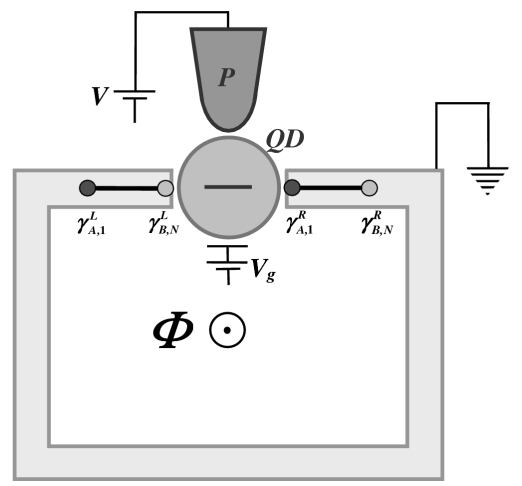

Fig. 1. Schematic of the device setup. The InAs quasi$1 \mathrm{D}$ semiconducting wires are in proximity with Al electrodes forming superconducting ring. The quantum dot, which mediates the transport in the Josephson junction, is weakly coupled to a normal tunnel electrode, allowing to measure current tunneling into the dot and to the grounded superconductor.

In this case the current through the junction is mediated by the zero energy MBS in the gap. As a result, it is dominated by the single electron tunneling rather than Cooper pairs tunneling. The change of the tunneling charge $q=2 e \rightarrow e$ makes the junction "fractional" and yields the $4 \pi$ periodicity of the Josephson current $J \sim \sin (\Delta \phi / 2)$ and halving of the resonant frequency $\omega_{r}$. The latter feature results in the doubling of the Shapiro steps, which has been observed experimentally [9].

In the present work we investigate a phase-biased Josephson junction with a quantum dot (QD) inside. Because occupation number and phase are complementary operators, it is extremely important to preserve the junction parity during current-phase measurement [10]. If the parity were not conserved during measurement, the measured phase dependence of the current would be $2 \pi$ periodic as in ordinary Josephson junction [11]. We show that the gate voltage manipulation of the dot 
level position can "stabilize" the junction parity and demonstrate that both $4 \pi$ periodicity and half of conductance quantum can be observed in the transport through the dot.

\section{Experimental realization of $1 \mathrm{D}$ topological superconducting wires}

The main obstacle in realization of $p$-wave topological superconductivity in quasi-1D wires is that electrons possess spin. However, a proper combination of the strong spin-orbit interaction inside the wire with external Zeeman field can lift spin degeneracy and cause that electrons behave as effectively spinless. It is realized in semiconductor wires with strong Rashba field and large $g$ factor as InSb $[4,5]$ or InAs [6, 12] wires. Such wires are exposed to proximity with usual $s$-wave superconductor, which induces effective pairing of spinless electrons. The wire enters a topological phase when the Zeeman field is the largest energy scale $E_{z}>\left(\Delta^{2}+\mu^{2}\right)^{1 / 2}$, where $\Delta$ is induced superconducting gap and $\mu$ chemical potential in the wire. In the wire prepared in this way unpaired Majorana states should emerge at its the ends, provided that the wire is long enough to exclude hybridization between them. It has been first modeled by Kitaev chain [13].

Very recently Kitaev chain was realized by depositing ferromagnetic atomic chain of Fe atoms onto conventional $\mathrm{Pb}$ superconductor [14]. In this case the external magnetic field was not needed due to the inherent ferromagnetism of the structure, and the strong spin-orbit field is provided by the $\mathrm{Pb}$ substrate.

\section{The model and theoretical approach}

The model, depicted in Fig. 1, is composed of two topological superconducting wires (TSW) attached to a quantum dot. Because of the large Zeeman field needed for topological superconductivity to appear the dot can be considered as effectively non-interacting. At the ends of the wires unpaired MBS are formed. The states closer to the QD are coupled to each other through the dot forming fractional Josephson junction. Additionally, the dot is coupled to the normal metal tunnel probe allowing the measurement of the electron transport through it. The model is similar to the experimental setup by Chang et al. [15]. Magnetic field piercing the ring allows to control the difference of superconducting phases between two sides of the junction.

The TSW are modelled by Kitaev chains [13] describing spinless $p$-wave superconductor. For the wire $\alpha=L, R$ :

$$
\begin{aligned}
H_{\alpha} & =-\mu_{\alpha} \sum_{i=1}^{N} c_{i \alpha}^{\dagger} c_{i \alpha} \\
& -\sum_{i=1}^{N-1}\left(t_{\alpha} c_{i \alpha}^{\dagger} c_{i+1 \alpha}+\left|\Delta_{\alpha}\right| \mathrm{e}^{\mathrm{i} \phi_{\alpha}} c_{i \alpha} c_{i+1 \alpha}+\text { H.c. }\right) .
\end{aligned}
$$

Further we assume the simplest topological phase; according to Kitaev for $\mu_{\alpha}=0,\left|\Delta_{\alpha}\right|=t_{\alpha} \equiv t$. In this regime, the Hamiltonian of the wires assumes the form

$$
H_{\alpha}=-t \sum_{i=1}^{N-1}\left(c_{i \alpha}^{\dagger} c_{i+1 \alpha}+\mathrm{e}^{\mathrm{i} \phi_{\alpha}} c_{i \alpha} c_{i+1 \alpha}+\text { H.c. }\right) \text {. }
$$

The induced superconducting order parameter, $\Delta$, inside the wires is chosen as a unit in our calculations. It is of the order of $0.3 \mathrm{meV}[4,5]$.

The Dirac fermions in the Kitaev chains can be expressed in terms of two Majorana fermions. For each $i$-site of the wire $\alpha$ :

$$
c_{i \alpha}=\mathrm{e}^{-\mathrm{i} \phi_{\alpha} / 2} \frac{1}{2}\left(\gamma_{B, i}^{\alpha}+\mathrm{i} \gamma_{A, i}^{\alpha}\right)
$$

We choose the gauge such that $\phi_{L} \equiv 0$ and $\phi_{R} \equiv \Delta \phi$, being the phase difference between wires $R$ and $L$.

The TSW Hamiltonian in this notation describes the coupling between MBS of adjacent sites

$$
H_{\alpha}=-\mathrm{i} t \sum_{i=1}^{N-1} \gamma_{B, i}^{\alpha} \gamma_{A, i+1}^{\alpha}
$$

and two end-site MBS $\gamma_{A, 1}^{\alpha}$ and $\gamma_{B, N}^{\alpha}$ in the wire $\alpha$ remain unpaired. Upon refermionization by the introduction of the Dirac fermions composed of the Majorana fermions of adjacent sites

$$
f_{\alpha i}=(1 / 2)\left(\gamma_{A, i+1}^{\alpha}+\mathrm{i} \gamma_{B, i}^{\alpha}\right),
$$

the wire Hamiltonian becomes diagonal

$$
H_{\alpha}=t \sum_{i=1}^{N-1}\left(2 f_{\alpha i}^{\dagger} f_{\alpha i}-1\right) \text {. }
$$

$H_{\text {tun }}$ describes tunneling of electrons between Left and Right wire and the dot

$$
H_{\text {tun }}=-t_{L} c_{N L}^{\dagger} d-t_{R} c_{1 R}^{\dagger} d+\text { H.c. }
$$

The dot level $\epsilon_{d}$ is coherently coupled to the end-wire MBS: $\gamma_{B, N}^{L}$ and $\gamma_{A, 1}^{R}$, which are converted into the Dirac fermion $d$ inside the dot. Thus, all the information, in particular on the superconducting phase difference, is encoded in the Dirac fermion $d$ in the dot

$$
d=\frac{1}{2}\left(\gamma_{A, 1}^{R}+\mathrm{i} \gamma_{B, N}^{L}\right) .
$$

It can be opposed to the process of electron tunneling from the dot into a single Majorana end state [16], in which this conversion does not take place. Taking into account Eqs. (3) and (8), the tunneling Hamiltonian is written in terms of the Majorana states revealing its dependences on superconducting phase

$$
\begin{aligned}
& H_{\text {tun }}=-\frac{\mathrm{i}}{2} t_{L}\left(\gamma_{A, 1}^{R} \gamma_{B, N}^{L}-\gamma_{B, N}^{L} \gamma_{A, N}^{L}\right)-\frac{\mathrm{i}}{2} t_{R} \\
& \quad \times\left[\sin \left(\frac{\Delta \phi}{2}\right) \gamma_{B, 1}^{R} \gamma_{B, N}^{L}\right. \\
& \left.\quad+\cos \left(\frac{\Delta \phi}{2}\right)\left(\gamma_{A, 1}^{R} \gamma_{B, N}^{L}-\gamma_{B, 1}^{R} \gamma_{A, 1}^{R}\right)\right] .
\end{aligned}
$$

In the last step, we refermionize $H_{\text {tun }}$ using Eqs. (5) and (8) and get

$$
\begin{aligned}
& H_{\text {tun }}=-\left(t_{L}+t_{R}\right) \cos \left(\frac{\Delta \phi}{2}\right)\left(d^{\dagger} d-\frac{1}{2}\right) \\
& \quad-\frac{t_{L}}{2}\left(f_{L N-1}^{\dagger} d^{\dagger}-f_{L N-1}^{\dagger} d+\text { H.c. }\right) \\
& -\frac{t_{R}}{2}\left[\mathrm{e}^{-\mathrm{i}(\Delta \phi / 2)}\left(f_{R 1}^{\dagger} d^{\dagger}+d^{\dagger} f_{R 1}\right)+\text { H.c. }\right] .
\end{aligned}
$$

When the topological wires are short, there is finite probability of hybridization of the end-side MBS in the each 
wire. It is described by the overlap $\epsilon_{\alpha} \sim \mathrm{e}^{-L_{\alpha} / \xi}, \xi$ is the superconducting coherence length. For the wire length $L_{\alpha} \sim 500 \mathrm{~nm}[4,15]$ it was negligible. The hybridization between end Majorana states hinders their detection and can be detrimental for logical operations on the Majorana states [17]. We show in our setup that for finite hybridization there are still signatures of the Majorana existence sensed by the tunnel probe coupled to the dot. Hamiltonian describing the process of hybridization has the form

$$
H_{h y b}=-\sum_{\alpha=L, R} \mathrm{i} \frac{\epsilon_{\alpha}}{2} \gamma_{A, 1}^{\alpha} \gamma_{B, N}^{\alpha} .
$$

As a result, a non-local Dirac fermion can be created out of two MBS in each wire: $f_{\alpha}=(1 / 2)\left(\gamma_{A, 1}^{\alpha}+\mathrm{i} \gamma_{B, N}^{\alpha}\right)$. Taking into account this relation and Eq. $(8), H_{h y b}$ can be written in terms of the Dirac fermions

$$
\begin{gathered}
H_{h y b}=\frac{\epsilon_{L}}{2}\left(f_{L}^{\dagger} d^{\dagger}-f_{L}^{\dagger} d+\text { h.c. }\right) \\
-\frac{\epsilon_{R}}{2}\left(f_{R}^{\dagger} d^{\dagger}+f_{R}^{\dagger} d+\text { H.c. }\right)
\end{gathered}
$$

Finally, the Hamiltonian of the dot reads

$$
H_{Q D}=\epsilon_{d} d^{\dagger} d
$$

where QD's level can be shifted by gate voltage with respect to the Fermi level in the probe, positioned at zero energy, $\epsilon_{F}=0$. The Hamiltonian of the normal tunnel probe to which the dot is attached reads

$$
H_{\text {probe }}=\sum_{k} \epsilon_{k, p} c_{k, p}^{\dagger} c_{k, p},
$$

and the Hamiltonian describing the dot-probe coupling has the form

$$
H_{Q D-\text { probe }}=\sum_{k}\left(t_{p} c_{k, p}^{\dagger} d+\text { H.c. }\right) \text {. }
$$

The total Hamiltonian written in terms of the Dirac fermions composed of Eqs. (6), (10), (12)-(15) is utilized to calculate retarded QD Green function, $G\left(t-t^{\prime}\right)=$ $-i \theta\left(t-t^{\prime}\right)\left\langle d(t) d^{\dagger}\left(t^{\prime}\right)+d^{\dagger}\left(t^{\prime}\right) d(t)\right\rangle$, by means of equation of motion method. The Hamiltonian is quadratic in creation and annihilation operators, which allows to obtain the QD Green function exactly. In energy domain it reads

$$
\begin{aligned}
& G_{d o t}^{-1}(\omega)=\omega-\epsilon_{d-}+\frac{\mathrm{i}}{2} \Gamma_{p}-\frac{1}{\omega+\epsilon_{d+}+\frac{\mathrm{i}}{2} \Gamma_{p}} \\
& \quad \times\left\{\frac{\omega^{2}\left[t_{L}^{4}+t_{R}^{4}-2\left(t_{L} t_{R}\right)^{2} \cos (2 \Delta \phi)\right]}{4\left(\omega^{2}-4 t^{2}\right)^{2}}\right. \\
& \left.\quad-\frac{\left(\epsilon_{R}^{2}-\epsilon_{L}^{2}\right)\left(t_{L}^{2}-t_{R}^{2}\right) \cos (\Delta \phi)}{2\left(\omega^{2}-4 t^{2}\right)}+\frac{\left(\epsilon_{R}^{2}-\epsilon_{L}^{2}\right)^{2}}{4 \omega^{2}}\right\}, \\
& \epsilon_{d-}=\epsilon_{d}-\left(t_{L}+t_{R}\right) \cos \left(\frac{\Delta \phi}{2}\right)+\frac{\omega\left(t_{L}^{2}+t_{R}^{2}\right)}{2\left(\omega^{2}-4 t^{2}\right)} \\
& +\frac{\epsilon_{R}^{2}+\epsilon_{L}^{2}}{2 \omega}, \\
& \epsilon_{d+}=\epsilon_{d}-\left(t_{L}+t_{R}\right) \cos \left(\frac{\Delta \phi}{2}\right)-\frac{\omega\left(t_{L}^{2}+t_{R}^{2}\right)}{2\left(\omega^{2}-4 t^{2}\right)} \\
& -\frac{\epsilon_{R}^{2}+\epsilon_{L}^{2}}{2 \omega},
\end{aligned}
$$

where $\Gamma_{p}=2 \pi t_{p}^{2} \rho_{p}\left(\epsilon_{F}\right)$ is the level broadening caused by the coupling of the dot to the tunnel probe. Having obtained $G_{d o t}(\omega)$, zero-bias conductance, $V \rightarrow 0$, can be calculated in the Meir-Wingreen [18] approach through the relation $\rho_{\text {dot }}(\omega)=-(1 / \pi) \Im G_{d o t}(\omega)$ :

$$
\mathcal{G}=\frac{\mathrm{e}^{2}}{h} \pi \Gamma_{p} \int d \epsilon\left(-\frac{\partial f(\epsilon)}{\partial \epsilon}\right) \rho_{d o t}(\epsilon)
$$

We assumed that the dot is weakly coupled to the normal probe, such that the entire voltage drop takes place in the junction between the dot and the probe.

\section{Numerical results and discussion}

In Fig. 2, the evolution of the spectral density of the dot for various possibilities of MBS states hybridizations inside the wires is shown. When both wires are long enough that the hybridization of the Majorana states at the ends of each wire is negligible, the Majorana $\gamma_{B, N}^{L}$ and $\gamma_{A, 1}^{R}$ compose the Dirac $d$ fermion inside the dot. In this case the density of states of the dot (solid curve) displays a peak centered at $\epsilon_{d}$ position. The picture changes when one of the wires is so short that the hybridization between MBS at it ends cannot be neglected (dashed line). Due to the finite $\epsilon_{L}$ hybridization between the Majorana $\gamma_{A, 1}^{L}$ and $\gamma_{B, N}^{L}$, an extended fermion $f_{L}$ is formed, and, in turn, the composite $d$ fermion is destroyed leaving the unpaired $\gamma_{A, 1}^{R}$ state. As a result, a characteristic Majorana peak at $\omega=\epsilon_{F}$ appears due to this unpaired state. This peak remains at its position independent of the position of $\epsilon_{d}$ or superconducting phase difference. Additionally, two peaks at $\omega \simeq \mp \epsilon_{L}$ can be observed, corresponding to the energy of the extended Dirac fermionic state $f_{L}$. Similar features can be observed for $\epsilon_{R} \neq 0$ and $\epsilon_{L}=0$ (not shown).

When both topological wires are short, that $\epsilon_{L} \neq 0$ and $\epsilon_{R} \neq 0$, two extended fermionic states $f_{L}$ and $f_{R}$ are formed and no unpaired Majorana state is left. As a result (dotted curve), the Majorana zero state disappears leaving the value of the density of states exactly zero at Fermi level, independent on the position of $\epsilon_{d}$ or phase. Instead, four peaks appear at $\omega \simeq \mp \epsilon_{L}$ and $\omega \simeq \mp \epsilon_{R}$ corresponding to energy of fermionic states formed out of Majoranas in the left and the right topological wires.

It is worth to emphasize that independent of the MBS hybridization scenario the density of states of the dot exhibits $4 \pi$ periodicity with respect to $\Delta \phi$, characteristic to fractional Josephson junction. It is reflected in zerobias conductance through the dot, as shown below.

In Fig. 3, the dependence of the finite temperature zero-bias conductance through the dot on the superconducting phase difference is presented. The dependences are shown for three different positions of the QD level and $\epsilon_{L} \neq 0$ and $\epsilon_{R}=0$. In this case an unpaired MBS in the $R$-wire produces a resonance at Fermi level. Each time the renormalized QD level reaches Fermi level, a peak in conductance is developed reaching its maximal value of the half-conductance quantum, $\mathrm{e}^{2} / 2 h$, characteristic for the existence of zero energy MBS. It is important to note that zero-bias conductance dependences reflect different junction parities; odd for $\epsilon_{d}=-0.1$, well below the Fermi 


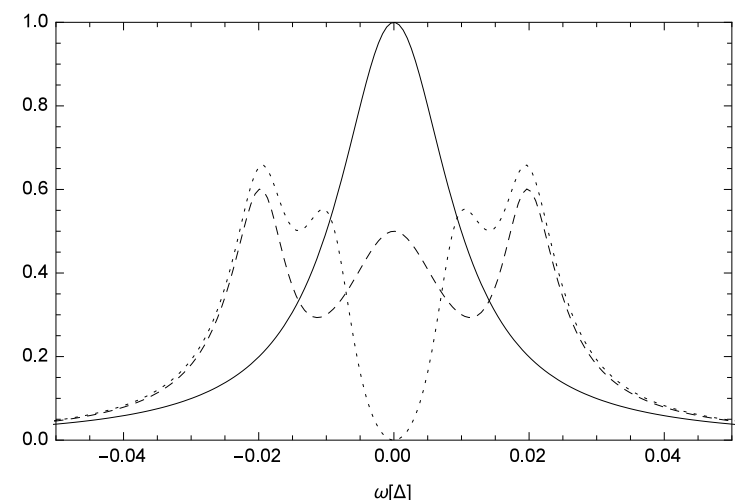

Fig. 2. Spectral density of the dot multiplied by $\gamma=$ $\pi \Gamma_{p}$ calculated for various overlap scenarios of the Majorana states inside the wires: $\epsilon_{L}=\epsilon_{R}=0$-solid, $\epsilon_{L}=0.02$ and $\epsilon_{R}=0$-dashed, $\epsilon_{L}=0.02$ and $\epsilon_{R}=0.01$ dotted. Other calculations parameters are: $\epsilon_{d}=0$, $t_{L}=t_{R}=0.1, \Gamma_{p}=0.02$ and $\Delta \phi=\pi$.

energy, and even in the opposite case of $\epsilon_{d}=0.1$. They possess fractional $4 \pi$ periodicity. It can be contrasted to the case of $\epsilon_{d}=0$ (solid curve) when the parity is not well defined and the junction displays $2 \pi$ periodicity as in a standard Josephson junction. Thus, the preservation of parity can be stabilized by gate voltage manipulation of the QD level position. It has crucial importance to the Majorana state detection inside the junction.

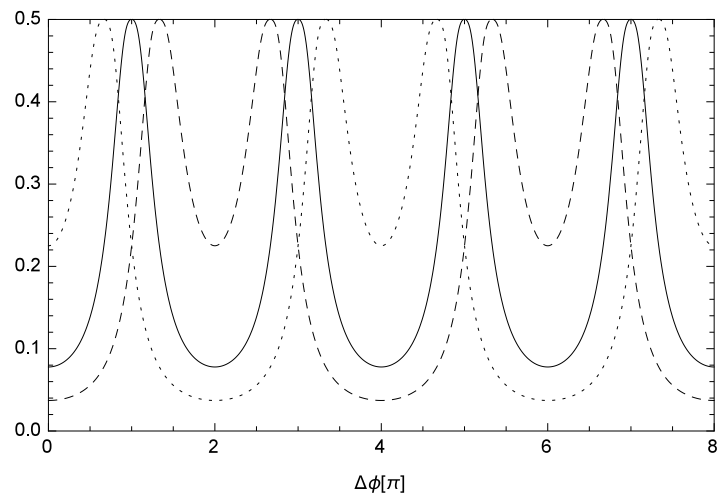

Fig. 3. Zero-bias conductance through the dot vs. superconducting phase difference calculated for $\epsilon_{L}=0.02$, $\epsilon_{R}=0$ and various quantum dot level positions: $\epsilon_{d}=0$ — solid, $\epsilon_{d}=-0.1$ - dashed and $\epsilon_{d}=0.1-$ dotted. Other calculation parameters are: $T=0.001$, $t_{L}=t_{R}=0.1, \Gamma_{p}=0.04$.

For the other scenarios of inside-wire hybridizations, i.e. for $\epsilon_{L}=0$ and $\epsilon_{R}=0$ or $\epsilon_{L} \neq 0$ and $\epsilon_{R} \neq 0$, the $4 \pi$ periodicity is also observed when $\left|\epsilon_{d}\right| \gg \epsilon_{F}$. In the former case when fermionic $d$ state is formed out of two end
Majorana from different wires, the zero-bias conductance reaches one conductance quantum, $\mathrm{e}^{2} / h$, when renormalized QD level crosses Fermi level. In the latter case the finite temperature conductance is very small (note that in $T=0$ the conductance is exactly zero independent of $\epsilon_{d}$ or $\Delta \phi$ because $\rho_{d o t}\left(\epsilon_{F}\right)=0$ in this case, see Fig. 2).

\section{Acknowledgments}

This work has been supported by the National Science Centre under the contract DEC-2012/05/B/ST3/03208.

\section{References}

[1] E. Majorana, Nuovo Cim. 14, 171 (1937).

[2] S. Das Sarma, M. Freedman, C. Nayak, NPJ Quant. Inform. 1, 15001 (2015).

[3] L. Fu, C.L. Kane, Phys. Rev. Lett. 100, 096407 (2008).

[4] V. Mourik, K. Zuo, S.M. Vrolov, S.R. Plissard, E.P.A.M. Bakkers, L.P. Kouwenhoven, Science $\mathbf{3 3 6}$ 1003 (2012).

[5] M.T. Deng, C.L. Yu, G.Y. Huang, M. Larsson, P. Caroff, H.Q. Xu, Nano Lett. 12, 6414 (2012).

[6] A. Das, Y. Ronen, Y. Most, Y. Oreg, M. Heiblum, H. Shtrikman, Nat. Phys. 8, 887 (2012).

[7] E.J.H. Lee, X. Jiang, R. Aguado, G. Katsaros, C.M. Lieber, S. De Franceschi, Phys. Rev. Lett. 109, 186802 (2012).

[8] H.O.H. Churchill, V. Fatemi, K. Grove-Rasmussen, M.T. Deng, P. Caroff, H.Q. Xu, C.M. Marcus, Phys. Rev. B 87, 241401(R) (2013).

[9] L.P. Rokhinson, X. Liu, J.K. Furdyna, Nat. Phys. 8, 795 (2012).

[10] A.M. Zagoskin, Quantum Theory of Many-Body Systems, Springer-Verlag, New York 1998.

[11] H.-J. Kwon, K. Sengupta, V.M. Yakovenko, Eur. Phys. J. B 37, 349 (2004).

[12] A.D.K. Finck, D.J. Van Harligen, P.K. Mohseni, K. Jung, X. Li, Phys. Rev. Lett. 110, 126406 (2013).

[13] A. Kitaev, Phys. Usp. 44, 131 (2001).

[14] S. Nadj-Perge, I.J. Drozdov, J. Li, H. Chen, S. Jeon, J. Seo, A.H. MacDonald, B.A. Bernevig, A. Jazdani, Science 346, 602 (2014).

[15] W. Chang, V.E. Manucharyan, T.S. Jespersen, J. Nygard, C.M. Marcus, Phys. Rev. Lett. 110, 217005 (2013).

[16] D.E. Liu, H.U. Baranger, Phys Rev. B 84, 201308(R) (2011).

[17] J. Alicea, Y. Oreg, G. Refael, F. von Oppen, M.P.A. Fisher, Nat. Phys. 7, 412 (2011).

[18] Y. Meir, N.S. Wingreen, Phys. Rev. Lett. 68, 2512 (1992). 